\title{
The Impact of Machine Learning on Modern Day Industries
}

\author{
J Qureshi
}

\begin{abstract}
Machine learning is more than just a buzzword. It is fundamentally changing the way that industries and the businesses within them carry out their everyday functions and activities from Finance and Recruitment right the way across to Sales and Marketing experience.

Machine learning can be defined as a subset of artificial intelligence (AI) that relies on models and inference to effectively perform a specific task, using algorithms and scientific models. In a more practical sense, a machine learning system takes a set of data and uses it to answer a question and continues to ingest more and more data to teach itself over time and ultimately become able to answer future questions in an unsupervised manner.

This paper explores how different industries and organizations are using machine learning algorithms in their day to day activities and where we see this transposed into our own lives.
\end{abstract}

Index Terms - machine learning, artificial intelligence, algorithm

The Impact of Machine Learning on Modern Day Industries

\section{WHAT IS MACHINE LEARNING?}

Machine learning (ML) is an application of artificial intelligence (AI). Some have considered it to be the best innovation since the microchip was invented. In essence, it is about training a machine to carry out the tasks a human would do and from that, continue to learn on its own without intervention. Computers do this by leveraging what we call Big Data. Big Data is a huge pool of data that can be processed rapidly and provide accurate responses. For example, conducting a Google search is a form of machine learning where input is entered into a system, analyzed and used to predict the most likely outcomes, just as an intelligent human would do.

Human really started seeing the sci-fi element of machine learning when voice controlled personal assistants like Amazon's Echo and Google Home were launched. These devices are now a standard in the home. Other fantasies like driverless cars are now not so distant in the future and already being run through trials.

There are several ways in which machine learning is being used, virtually spanning every conceivable industry to improve decision making, increase efficiency or better customer experience.

\section{NATURAl LANGUAGE PROCESSING}

Natural Language Processing (NLP) has become one of the most commonplace applications of machine learning over the last few years with technologies such as Amazon Alexa and Google Home taking the market by storm. In its purest form, NLP translates text or speech into data and over time, learns how human interact and provide recommendations or even decisions based on a vast amount of information. An everyday example that we all use is the autocomplete function on a mobile phone which is a fantastic example of unsupervised learning to create accurate recommendations.

\section{A. NLP Case study - Hello Barbie (Marr, 2018, p. 1)}

Using incredibly advanced machine learning technology, Hello Barbie is able to translate the speech of a child and respond with the correct answer from over 8,000 different possible combinations based on what the child says. The servers can transmit a response back in under 1 second. Beyond this, it even learns from its answers so as they can be recited later if asked again.

\section{INTERNET OF THINGS (IOT)}

The Internet of Things refers to a network of devices that are related or connected to each other via some sort of network. The most common application is perhaps in Smart Homes, where millions of properties now utilize the likes of Nest for intelligent thermostats or leakbots to detect water flow in pipes. All of these products are examples of machine learning where they continue to learn as they collect more and more data over time. Companies are increasing using machine learning to increase the value of their IoT projects

\section{A. IoT Case Study-predictive maintenance (Forrest, 2018)}

Machine learning algorithms can provide predictions for maintenance of complex systems, such as recommending when they might fail. This could be hugely beneficial to the construction image or large manufacturing plants for example. One example, which also utilizes Blockchain type technology, has been developed by IBM together with Walmart where this technology tracks the temperature and storage of food all the way from its source to the shelf. If at any point it drops below or above a specified temperature, the algorithm flags it and the Blockchain is broken. With this, the food never makes the shelf and averts the risk of food poisoning that currently affects 1 in 10 people each year.

\section{CUSTOMER EXPERIENCE}

There are several ways in which machine learning technology has the capacity to improve customer experience. In a highly digital world, it is important for businesses to 
provide customers with as many ways as possible to communicate with them but with each of these methods comes challenges. Every single person wants a different kind of interaction and it simply isn't possible to have enough human resource to account for every eventuality. This is where machine learning comes in. Over the last few years, there has been intense investment in the likes of ChatBots, recommender systems (Netflix or Spotify) and automated supply chain management or stock control. Each of these comes with a goal of creating the best possible experience for each individual customer.

\section{A. Customer Experience Cast Study - Spotify}

Spotify use data from over 100 million customers to create amazing advertising campaigns which humanized machine learning. The ad campaigns featured slogans such as "Dear person who played 'Sorry' 42 times on Valentine's Day, what did you do?" This took away some of the fearmongering that has gone around about how companies use data and given it that human element. Beyond this, Spotify members are provided with automatic playlists of music recommended to then by the platform using their data. Again, as Spotify learns what each customer listens to (the machine learning part), they can create a very specific experience for every single individual who uses the application. Netflix follows a very similar recommender style process through the shows and movies that it recommends to its users.

\section{IMAGE RECOGNITION (HTTPS://DZONE.COM/, N.D.)}

A lot of recent technological innovations seem to be focusing on image or facial recognition. The most common application is logging into a smartphone or financial applications. This type of technology uses a more complex form of machine learning known as deep learning. Whilst machine learning is able to come to conclusions about the data it uses, deep learning attempts to work out whether those conclusions are actually correct, given enough time to work it out. This process is very important for image recognition for something like a driverless car to work out the difference between a pedestrian and a signpost perhaps.

\section{A. Image Recognition Case Study - Facebook (https://www.marutitech.com/, n.d.)}

Facebook is now able to carry out face recognition at an amazing $98 \%$ accuracy which is comparable to the ability of humans. This is most noticeable with how it tags pictures, recognizing you, your friends or family almost instantly in most cases through its deep learning networks. The methods used to do this are complex but in its most basic of senses, the deep learning platform takes an image and classifies different parts of it, converting it into data and matching that to other similar datasets. To be entirely accurate, the algorithm needs a huge amount of data, which is something Facebook now possesses, hence its ability with tagging the correct people. Let's say we wanted to train a machine to work out if a picture was of a cat. We might start by loading it with millions of pictures of cats and millions which are not cats. Over time, it will take all of that data, decide on the set of rules required akin to a human brain and tell you whether or not an image is in fact a cat.

\section{Medical Diagnosis (SenNaAR, 2019)}

Medical diagnostics are a set of procedures designed to detect infections, conditions and disease. AI and machine learning technology is starting to play an integral role in the medical industry and already has a number of applications. The main reason for the industry focus on machine learning is that errors account for as much as $10 \%$ of patient deaths, not through any malpractice of any kind but due to the inability of the human brain to calculate billions and billions of data attributes like a machine has the capacity to do. Ultimately, national health expenditure is estimated to have reached up to \$3.4 trillion in 2016 (Sennaar, 2019) and some investment is required to reign this back in.

\section{A. Medical Diagnosis Case Study - Stanford University (Stanford Unviersity, 2017)}

Stanford University has created a deep learning algorithm capable of diagnosing skin cancer in patients which is at least as accurate as human diagnosis. The algorithm used over 130,000 images of skin lesions that represented over 2,000 diseases. With early detection accounting for a $97 \%$ five year survival rate, this is a huge leap forward for the medical profession and ultimately human life itself.

Stanford's testing compared the algorithm to 21 different dermatologists and the results had the same level of accuracy. The time and resource savings of those professionals will be huge going forwards if it is possible to go into full deployment with such technology.

\section{FINANCIAL SERVICES}

Financial services is typically or at least stereotypically quite old fashioned but more and more organizations in the industry are embarking on machine learning and AI projects having recognized the income potential and cost savings from its implementation. Most financial institutions have a vast amount of data having been established for many years and the bigger challenge comes in ensuring the data is clean, de-duped and classified before they start any machine learning project. However, if they start to consider the potential for increased efficiency, reduced operational costs, improving income and better security, financial service providers will soon start to see that the benefits of investment heavily outweigh the cons.

\section{A. Financial Services Case Study-JP Morgan (Didur, 2018)}

JP Morgan launched a Contact Intelligence platform that uses Natural Language Processing as one of its machine learning techniques. The concept of this system is to process legal documents and extract data from them. Previously, manual reviewing 12,000 credit documents would have taken something like 360,000 labor hours whereas the machine learning algorithm was able to reduce this to just 2 or 3 hours. A common myth is that this leads to mass unemployment but actually, the company are able to simply deploy their staff into more useful areas such as reviewing the data produced and working on root causes and solutions for example. 


\section{PREDICTION}

Machine learning is now being favored over predictive analytics as a method of making business decisions. Traditionally, analysts would take a set of existing data and use statistical models to work out the patterns that lie within in and feed that back into different teams or departments to come up with potential solutions. Machine learning on the other hand looks forwards and uses complex algorithms to predict what is the most likely outcome in the future. For example, we see every day is banks trying to assess the potential debtor risk. Using millions or billions of variables from existing data, the machine learning algorithm will reach a decision as to whether each person is a good or bad risk, maybe based on elements such as age, income, job type and credit history and matching that to previous experiences.

\section{A. Prediction Case Study (Brock, 2016)}

Companies are taking advantage of their own data to predict how customers purchase or need customer support before the events even happen. A startup company had 2,000 users signing up for a 10-day free trial of their product but only $3 \%$ were registering for the paid subscription (Brock, 2016). The challenge was increasing that conversion rate to improve income and finding out what the triggers were to ensure customers moved to the paid subscription.

For this challenge a prediction engine was built that had the capability of working out which users are likely to convert in real-time. Using this information, the support staff were able to focus on the trial users with a high likelihood of converting and offer less resource to those that were only in it for the free trial. With this and some other predictive algorithms in place, the startup were able to increase their conversion rate from $3 \%$ to $7.4 \%$ which is huge for any company just setting out.

\section{RECOMMENDER SYSTEMS}

In the digital world, customers don't like to make too many choices and many retailers and moving to recommender style systems that reduce the number of questions a customer needs to answer. In data terms, a recommender system works by using data to assign a score to each item based on how it predicts the user would score it. In some of the smarter systems, this means it can tell a customer what they want before they even know they want it. If you consider Google, which most of us use every day, the predictive search is a recommender system and sometimes just by typing a few letters, it is now able to work exactly what you are looking for.

\section{A. Recommender System Case Study-Netflix (Plummer, 2017)}

Arguably the most popular recommender system of recent times is Netflix. The algorithm works by splitting users into several groups, somewhere around 2000 and whichever group you are in, dictates the type of show recommendations that you receive. If you compare your account to that of your friend, the chances are they would look completely different. The machine learning algorithms direct users to shows they would probably have never previously chosen as in general; human are creatures of habit. The smartest part is that the algorithms mainly use implicit data rather than explicit data.
For example, you might not have told Netflix that you hate watching horror movies but the data shows that you watch them regularly and switch off half way through, so it stops recommending them. If your behavior changes, it will react accordingly.

\section{Contract Review}

Lawyers are very busy and automating their day-to-day activities could be ground-breaking for the industry. If they have more time to focus on the case at hand rather than doing manual research and admin work, productivity levels will soar. Machine learning brings the ability to analyze vast volumes of documents in minutes meaning lawyers don't have to sift through books and papers to get the required information; cases that take months or years to resolve could ultimately be reduced to weeks or days .

\section{A. Contract Review Case Study (LawGeex, February 2018)}

Legal tech startup LawGeex published a study demonstrating their artificial intelligence software is able to analyze contracts such as Non-Disclosure Agreements (NDAs) faster and more accurately than even the most experienced of lawyers. The aim of the trial was to find any issues within the agreements.

The study used fie legal contracts which were reviewed by the LawGeex software and 20 experienced lawyers to find issues related to risk. The results were that the AI solution identified the specific legal issues at a 94\% accuracy against only $85 \%$ for the lawyers. In fact, the best lawyer was only able to equal the ability of the LawGeex software whilst the lowest performing only achieved $67 \%$ accuracy.

\section{OTHER APPLICATIONS}

There are of course many other applications of machine learning but this paper has focused on some of the key developments over the last two years. Financial trading, marketing personalization, fraud detection and the construction industry to name a few are all going through a period of heavy AI and machine learning investment and will also continue to grow in the foreseeable future, changing the way we go about everyday life. It is also important to consider the impact of Virtual and Augmented Reality in these industries as they continue to have a resurgence.

\section{SUMMARY}

Machine learning is having a huge impact on modern day industry and everyday living which will only continue to grow as we become more digitally transformed. IBM (2018) are predicting that machine learning will ultimately become part of every single software platform that we use and be expected as standard rather than a novelty. As algorithms continue to re-train through the addition of data, they will become smarter, possibly to a point where they even seem like humans but without the consciousness. All in all, it is important to embrace AI and machine learning technology, no matter what industry you are in. 
The Impact of Machine Learning on Modern Day Industries

\section{REFERENCE}

[1] (n.d.). Retrieved from https://dzone.com/.

[2] (n.d.). Retrieved from https://www.marutitech.com/.

[3] Brock. (2016). Retrieved from Predicting Customer Behaviour.

[4] Didur. (2018). Towards Data Science. Retrieved from https://towardsdatascience.com.

[5] Forrest. (2018). How to use machine learning to accelerate your IoT initiatives.

[6] Marr. (2018). 27 Incredible Examples Of AI And Machine Learning In Practice.

[7] Plummer. (2017). This is how Netflix's top-secret recommendation system works. Retrieved from www.wired.co.uk.

[8] Williams. (2019). 10 Ways Machine Learning Will Change Our Lives Retrieved from Towards Data Science

[9] Sennaar. (2019). Machine Learning for Medical Diagnostics - 4 Current Applications.

[10] Stanford Unviersity. (2017). Retrieved from https://news.stanford.edu/2017/01/25/artificial-intelligence-used-ide ntify-skin-cancer/

[11] Shekhar, Sarmah Simanta. "Artificial Intelligence in Automation." Artificial Intelligence (2019).

[12] Michie, D., Spiegelhalter, D. J., \& Taylor, C. C. (1994). Machine learning. Neural and Statistical Classification, 13.

[13] Dietterich, Thomas G. "Machine-learning research." AI magazine 18.4 (1997): 97-97.

[14] Mohri, Mehryar, Afshin Rostamizadeh, and Ameet Talwalkar. Foundations of machine learning. MIT press, 2018 